\title{
Real time 3-dimensional transesophageal echocardiography is more specific than 2-dimensional TEE in the assessment of left atrial appendage thrombosis
}

\author{
Dan Marek, David Vindis, Eva Kocianova
}

Background. In patients indicated for detection of intraatrial thrombus $(T), 2$-dimensional transesophageal echocardiography (2DTEE) is routinely used but differentiation between T and trabeculae or artifacts in the left atrial appendage (LAA) is often difficult.

Aims. To compare the diagnostic value of real time 3D-transesophageal echocardiography (RT3DTEE) and 2DTEE in the assessment of LAA thrombosis.

Patients and Methods. One hundred and ten consecutive patients (73M, aged 64+-13) were examined by 2DTEE. In terms of possible T, individual pts were diagnosed as negative (N2), uncertain trabecular finding (U2), other/artifacts (O2), and clearly positive (T2). The RT3DTEE was then applied and the categorization repeated (N3,U3,O3 and T3, resp.). Finally, the operator decided whether the RT3DTEE. A: had an additional diagnostic value, and/or B: changed the definite diagnosis of thrombosis.

Results. N2:71; U2:17; O2:19; T2:3; N3:97; U3:1; O3:12;T3:0. Ad A/ RT3DTEE enabled us to refine or change the diagnosis in 26/110 cases. 17 pts were switched from group U2 to N3, 7:O2-N3 and 2:T2-N3. 12 pts from O2 and 1 pt from T2 remained unclarified. Ad B/ Diagnoses were changed in 6 patients after RT3DTEE. A very suspicious T2 was found in 3 patients. In 1, the finding was requalified definitely as musculi pectinati. In the second patient, the finding was recategorized as just a spontaneous echocontrast without T. In another 4 patients, T in LAA was definitely excluded after RT3DTEE (1 patient switched from U2 to N3, 3 from O2 to N3). In another 13, the findings remained unclear, mostly because of poor quality image. No real thrombus was found in this study.

Conclusion. RT3DTEE provides additional information, which may be helpful in the differentiation of thrombus from other findings. It is particularly useful in the identification of muscular trabeculae in the left atrial appendage.

Key words: left atrial appendage, thrombus, real time 3D echocardiography, musculi pectinati

Received: July 14, 2011; Accepted with revision: January 12, 2012; Available online: January 30, 2012

http://dx.doi.org/10.5507/bp.2012.012

Department of Internal Medicine I - Cardiology, Faculty of Medicine and Dentistry, Palacky University Olomouc, Czech Republic Corresponding author: Dan Marek, e-mail: dan.marek@fnol.cz

\section{INTRODUCTION}

Detection of intracardiac thrombus $(\mathrm{T})$ is an important issue particularly in patients with atrial fibrillation, after a stroke, in patients indicated for catheter interventions, etc. The LAA thrombus in particular represents a pathophysiological condition with a very high risk of potential cardioembolic event (a stroke or limb and organ embolism). Two-dimensional transesophageal echocardiography (2DTEE) is routinely used for that purpose. Visualization of the left atrial appendage (LAA) is particularly important, as it is a common site of thrombosis. It is difficult to see LAA on transthoracic echocardiography, but it is not a problem in most of the patients undergoing TEE examination. However, in some patients there may be difficulties in obtaining a good quality image of the LAA. Interposition of tissues, unusual heart position, breathing movements and other ultrasound artifacts may interfere with the imaging. In this case, it may be difficult even for an experienced echocardiographist to make a definite statement about the presence or absence of LAA thrombus. Also, differentiating between an orga- nized thrombus and $3 / 3$ spontaneous echocontrast may be tricky. The presence of musculi pectinati in LAA often complicates the diagnosis. Some people have very distinct muscular structures in the LAA, which is often multilobular. In some patients, musculi pectinati are almost missing (in very young or very old people) (ref. ${ }^{1,2}$ ). Muscular structures often mimic a thrombosis on TEE examination, particularly if a limited number of planes are used for assessment (Fig. 1, left side).

Real-time-three dimensional transesophageal echo (RT3DTEE) may provide additional information to facilitate the final decision in these patients. Only a few minor studies have been published on this topic, mainly in the form of case reports ${ }^{3-5}$. A typical LAA thrombus on RT3DTEE is seen in (Fig. 2).

The aim of this study was to compare the diagnostic value of RT3DTEE and 2DTEE in assessment of LAA thrombosis. 


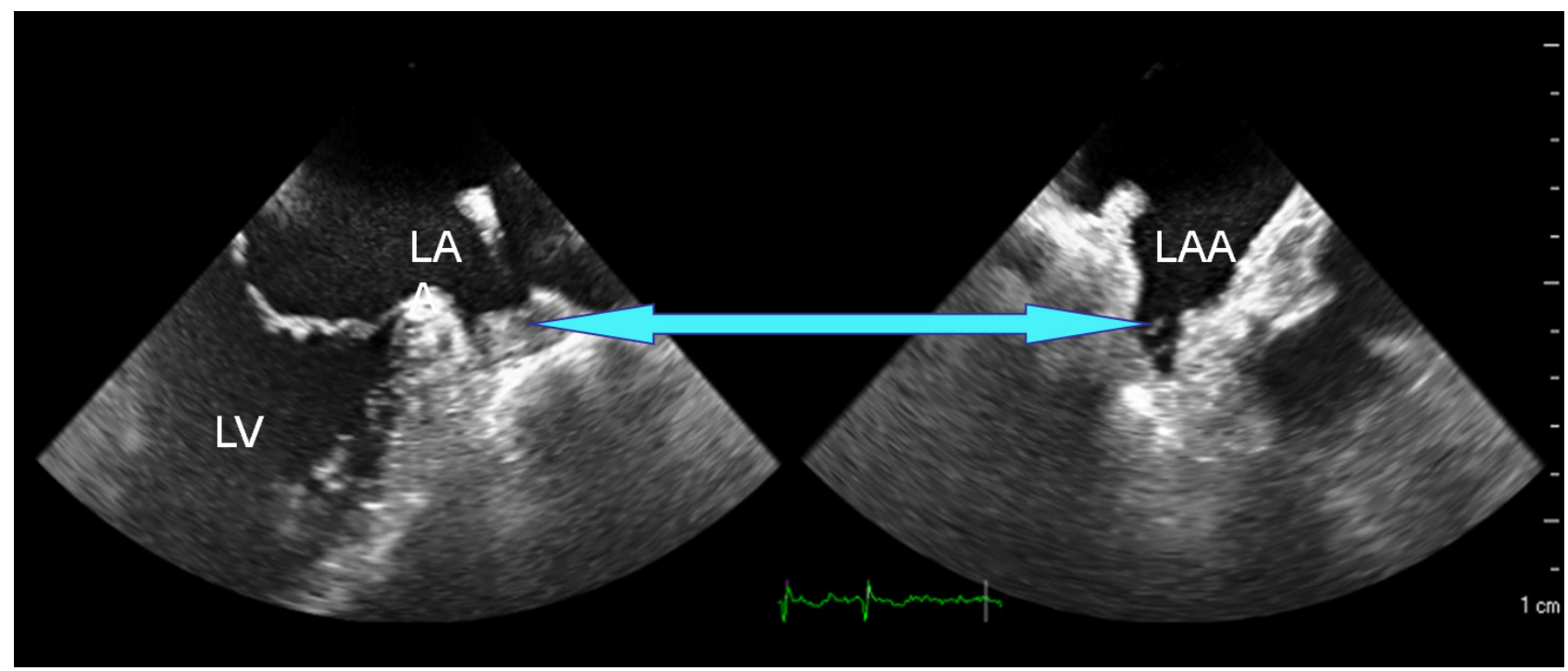

Fig. 1. Two perpendicular planes show the typical muscular structures in the LAA. On the left, the arrow depicts an echogenic structure, which may resemble an LAA thrombus. On the right, the corresponding echo belongs clearly to the musculi pectinati. LV - left ventricle LA - left atrium LAA - left atrial appendage

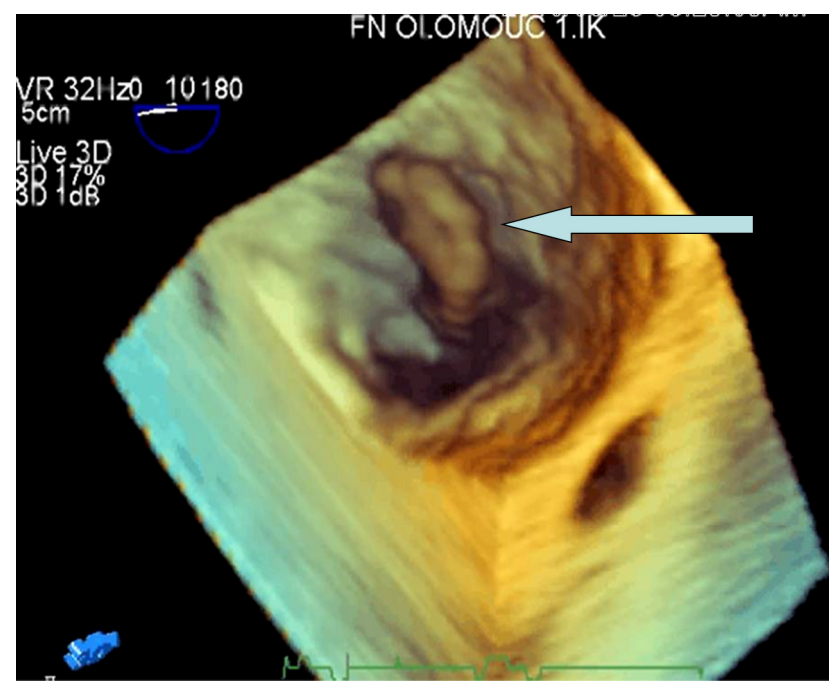

Fig. 2. Left atrial appendage orifice seen from semi en-face view. The thrombus is protruding from the appendage (arrow).

\section{METHODS}

One hundred and ten consecutive patients ( $73 \mathrm{M}$, aged 64+-13) were examined by 2DTEE for various reasons in the tertiary cardiology center, University Hospital Olomouc. Sixteen had persistent/chronic atrial fibrillation or flutter at the time of the TEE investigation. In another 12 patients, there was a paroxysmal atrial fibrillation or flutter in their histories. In each patient, a careful assessment of LAA was a routine part of the examination. Only patients with a good tolerability of the TEE examination (either under sedation or without it), which allowed a comfortable assessment of the LAA, were included.

Three experienced TEE operators conducted the

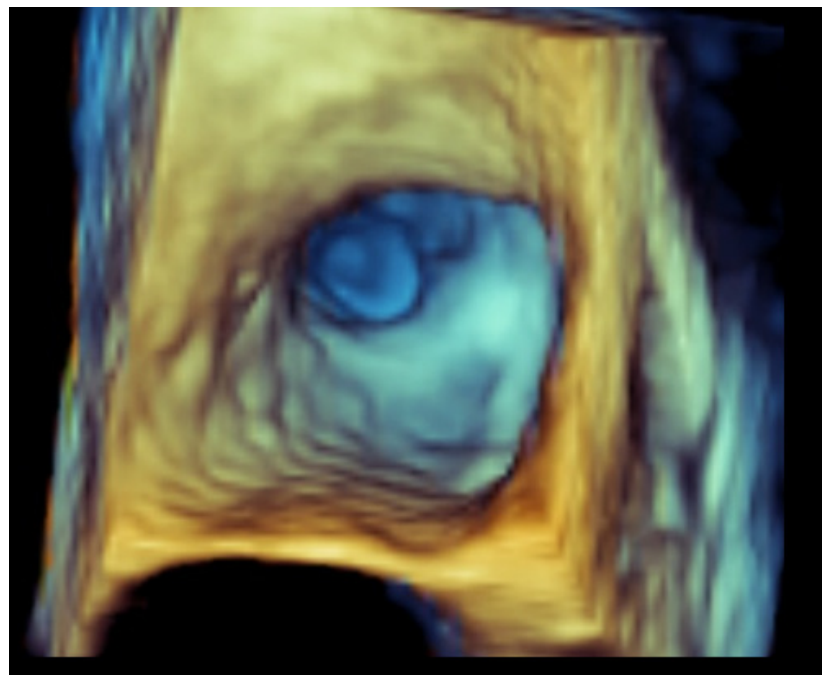

Fig. 3. Dilated LAA, seen from the en-face view. No transverse muscular structures are present.

study, each of them having more than 5 years' practice in LAA assessment by 2DTEE. Two operators had 11 months' experience with RT3DTEE; the third operator had a 6 months' experience with RT3DTEE. Philips "iE33" machine with $6 \mathrm{MHz}$ TEE probe was used for the examination in all cases.

First, routine 2DTEE was performed and the LAA was carefully scanned by multiplane TEE approach. Default machine settings (focus, zoom, frame rate, gain, compression etc.) were adjusted according to operator's discretion in order to improve the TEE image quality, if needed. In terms of the possible presence of thrombus in LAA, individual pts were diagnosed by 2DTEE and divided into 4 groups: negative - N2, uncertain trabecular finding - U2, 
other (poor image quality or possible artifacts) - $\mathrm{O} 2$, and clearly positive - T2. Patients who had a typical muscular structure in the LAA even on the 2DTEE were classified as N2. Patients with a spontaneous echocontrast without $\mathrm{T}$ were also classified as N2. Included in the U2 group were only those patients in whom the operator was not sure whether the structures were of physiological origin. The RT3DTEE was then applied and the categorization repeated (N3, U3, O3 and T3, respectively). Apart from the above mentioned pre- and post-processing machine adjustments, transverse X-plane, 3D zoom, full-volume with cropping and live 3D regimens were used according to the operator's discretion. Finally, it was assessed whether using RT3DTEE

A: was helpful and had an additional diagnostic value (i.e. number of pts where the unclear finding was either confirmed or requalified in terms of possible thrombosis: $\mathrm{U} 2$ to N3, U2 to T3, O2 to N3, T3), and/or

$\mathrm{B}$ : changed the definite diagnosis of LAA thrombosis.

\section{RESULTS}

Table 1 shows the classification of the ultrasound findings. Table 2 depicts the re-categorization of the findings.

Ad A/ RT3DTEE helped to refine or change the diagnosis in 26 cases (see Table 2).

Ad B/ Diagnoses were changed after RT3DTEE in 6 patients. A very suspicious T2 was found in 3 patients. In 1 , the finding was requalified definitely as musculi pectinati. In the second patient, the finding was re-categorized only as a spontaneous echocontrast without $\mathrm{T}$. (The last $\mathrm{T} 2$ patient remained unclear - O3). In 4 patients, a thrombus in LAA was excluded after RT3DTEE (1 switched from $\mathrm{U} 2$ to $\mathrm{N} 3,3$ from $\mathrm{O} 2$ to $\mathrm{N} 3$ ).

On the other hand, in another 13 pts, the findings remained unclear, mostly because of poor quality image. In the remaining patients, RT3DTEE was not helpful. No typical thrombus was found in this set of patients.

\section{DISCUSSION}

Differentiation between $\mathrm{T}$ and trabeculae/artifacts is often difficult, especially when a suspected echo is seen in the left atrial appendage (LAA). According to Agoston's team, 3D echo is more useful in LAA imaging than $2 \mathrm{D}$ echo. In their study, live $3 \mathrm{D}$ visualization was dependent on image quality, suboptimal in 100 and diagnostic in 104 patients. Overall, LAA was visualized in $93(45.5 \%)$ patients by 2DE compared to $139(68.1 \%)$ by 3DE $(P<0.0001)$. In 100 patients with suboptimal image quality by live 3DE, LAA visualization was $16 \%$ by $2 \mathrm{DE}$ and $35 \%$ by live 3DE, whereas in 104 patients with diagnostic images, LAA was visualized in 77 (74\%) by 2DE, and in all $104(100 \%)$ patients by 3DE $(P<0.0001)$. In 37 patients referred for TEE, 3DE visualized LAA in 34 patients with diagnostic image quality ${ }^{6}$.
Table 1. 2DTEE (upper row) and RT3DTEE (bottom row) findings.

\begin{tabular}{cccc}
\hline $\mathrm{N} 2: 71$ & $\mathrm{U} 2: 17$ & $\mathrm{O} 2: 19$ & $\mathrm{~T} 2: 3$ \\
\hline $\mathrm{N} 3: 97$ & $\mathrm{U} 3: 1$ & $\mathrm{O} 3: 12$ & $\mathrm{~T} 3: 0$ \\
\hline
\end{tabular}

Table 2. The arrows indicate the shift of patients between the groups (dark arrows: RT3DTEE helpful; light arrows: no additional value of RT3DTEE - thrombus could not have been excluded).

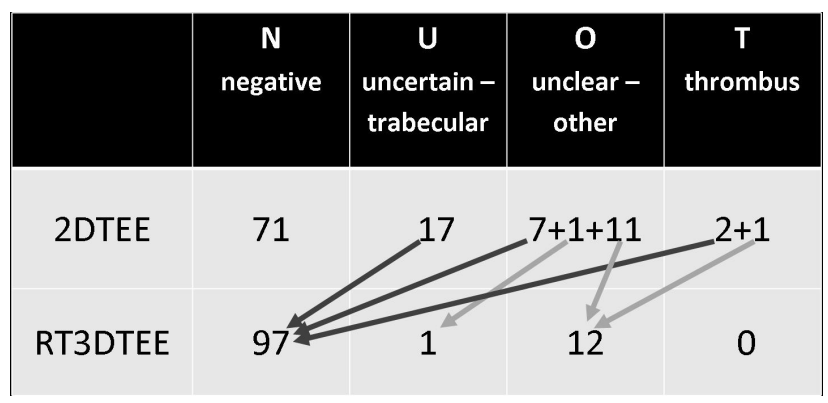

Some authors state that even a transthoracic 3DE may be more precise than 2DTEE in certain situations; 3DE revealed thrombi in the same patients as 2DTEE (7/92) and excluded thrombi in another 9 patients, where the findings on 2DTEE were suspicious of thrombi. In fact, muscular trabeculi mimicked the thrombi ${ }^{7}$.

Anwar's experience was similar: both 2DE and RT3DE detected 45 intracardiac thrombi and RT3DE could detect seven additional thrombi in five patients (three in the left atrial appendage and four in the left ventricular apex), showing, moreover, better interobserver agreement than 2DE. RT3DE was superior to 2DE in the assessment of thrombus mobility, differentiation between the thrombus and myocardium, and delineation of the changes in thrombi structure, e.g. calcification, degeneration, or lysis ${ }^{8}$. Transesophageal RT3DE was also better in the description of thrombi in Muller's study ${ }^{9}$. 2DE probably underestimates the dimensions of thrombi, compared to RT3DE, as shown by Asch $^{10}$.

Undoubtedly, RT3DTEE is a unique tool to look into the LAA orifice from the enface view, with a clear delineation of musculi pectinati. The huge variability in muscular structures in LAA is astonishing: in some patients the trabeculi are almost missing. In others, there is an extensive trabecular septation, creating a number of recessi in LAA (Fig. 3, 4).

In our experience, supported by this study, the differentiation between the muscular structure and other findings is probably the main asset of RT3DTEE in evaluation of intracardiac structures. Not only the above mentioned en-face view; even the transverse Xplane (2 perpendicular planes screened simultaneously) can help. This has also 


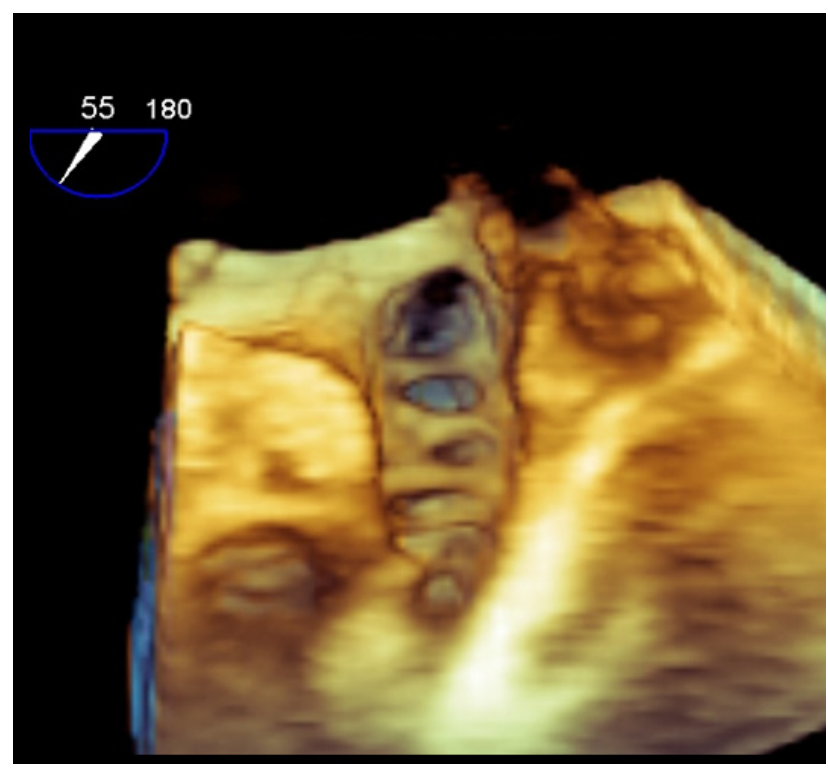

Fig. 4. Extremely trabecularized LAA (longitudinal section).

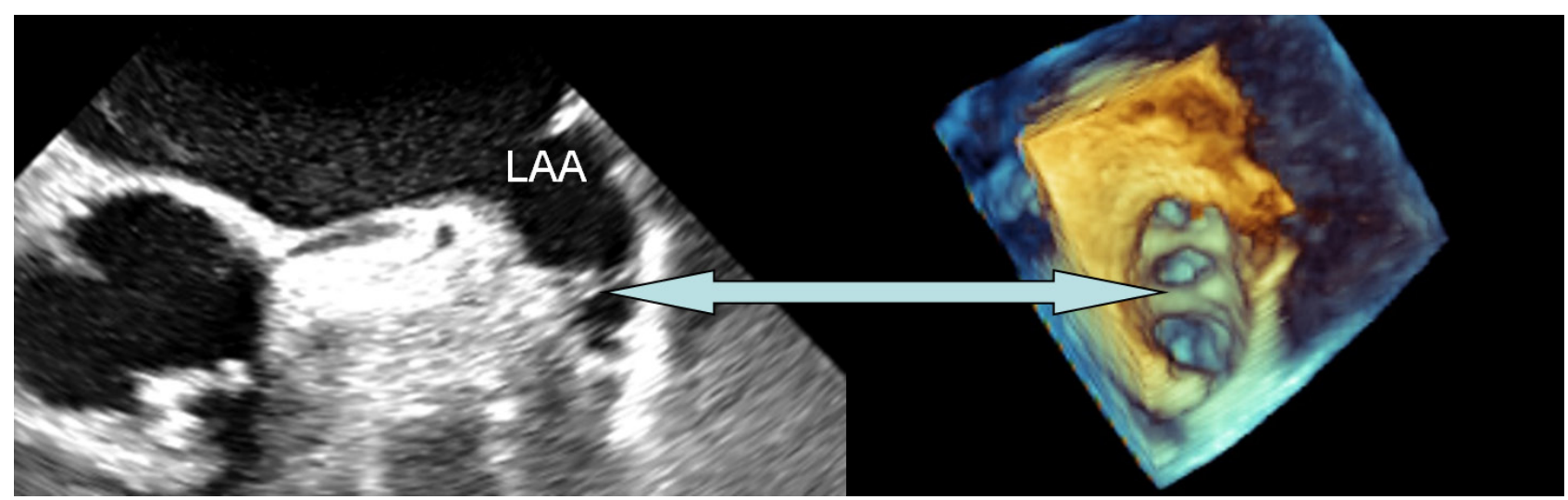

Fig. 5. Trabeculae (arrow) seen on 2DTEE (long axis) and RT3DTEE (en-face view). LAA - left atrial appendage.

been documented in other published case reports and small studies ${ }^{3-5}$. A typical picture of musculi pectinati seen in $2 \mathrm{DE}$ and $3 \mathrm{DE}$ is seen in (Fig. 5).

No wonder that having to rely in the ancient TEE era on just 1 or 2 planes made the operator's decision very difficult. Indeed, in some cases, these "structures" must have been mistaken for suspicious thrombi in some cases (see also Fig. 1).

Unfortunately, despite these facts, the RT3DTEE is not $100 \%$ sensitive or specific in thrombi identification either. The additional diagnostic value over the $2 \mathrm{DE}$ is also limited. RT3DTEE did not help in a substantial portion of patients in whom the 2DTEE was not diagnostic. The reason for this was definitely the poor image quality. In other words, if the 2DE image is not satisfactory, one cannot expect an excellent 3DE image, which - obviously - results from the technical limitations of emission, reception and processing of ultrasound signal. Such a "difficult patient" remains a challenge for the clinician, and a com- parison of RT3DTEE with other imaging methods in this group of patients will be of major interest.

\section{CONCLUSIONS}

RT3DTEE provides additional information, which can be helpful in differentiating a thrombus from other findings. In our study, real-time 3D transesophageal echocardiography showed greater specificity than twodimensional transesophageal echocardiography in the identification of thrombus in the left atrial appendage. It is particularly useful in the identification of muscular trabeculae in the left atrial appendage. Due to the physical parameters of ultrasound emission, reception and postprocessing, one can hardly expect a good 3D image if the two-dimensional image quality is not satisfactory. 


\section{REFERENCES}

1. Veinot JP, Harrity PJ, Gentile F, Khandheria BK, Bailey KR, Eickholt JT, Seward JB, Tajik AJ, Edwards WD. Anatomy of the normal left atrial appendage: a quantitative study of age-related changes in 500 autopsy hearts: implications for echocardiographic examination. Circulation 1997;96:3112-5.

2. Kerut EK. Anatomy of the left atrial appendage. Echocardiography 2008;25:669-73.

3. Latcu DG, Rinaldi JP, Saoudi N. Real-time three-dimensional transoesophageal echocardiography for diagnosis of left atrial appendage thrombus. Eur J Echocardiogr 2009;10:711-2.

4 .Mizuguchi KA, Burch TM, Bulwer BE, Fox AA, Rizzo RJ, Shernan SK. Thrombus or bilobar left atrial appendage? Diagnosis by real-time three-dimensional transesophageal echocardiography. Anesth Analg 2009;108:70-2.

5. Willens HJ, Qin JX, Keith K, Torres S. Diagnosis of a bilobed left atrial appendage and pectinate muscles mimicking thrombi on real-time 3-dimensional transesophageal echocardiography. J Ultrasound Med 2010;29:975-80.
6. Agoston I, Xie T, Tiller FL, Rahman AM, Ahmad M. Assessment of left atrial appendage by live three-dimensional echocardiography: early experience and comparison with transesophageal echocardiography. Echocardiography 2006;23:127-32.

7. Karakus G, Kodali V, Inamdar V, Nanda NC, Suwanjutah T, Pothineni KR. Comparative assessment of left atrial appendage by transesophageal and combined two- and three-dimensional transthoracic echocardiography. Echocardiography 2008;25:918-24.

8. Anwar AM, Nosir YF, Ajam A, Chamsi-Pasha H. Central role of realtime three-dimensional echocardiography in the assessment of intracardiac thrombi. Int J Cardiovasc Imaging 2010;26:519-26.

9. Muller S, Feuchtner G, Bonatti J, Laufer G, Hiemetzberger R, Pachinger O, Barbieri V, Bartel T. Value of transesophageal 3D echocardiography as an adjunct to conventional $2 \mathrm{D}$ imaging in preoperative evaluation of cardiac masses. Echocardiography 2008;25:624-31.

10. Asch FM, Bieganski SP, Panza JA, Weissman NJ. Real-time 3-dimensional echocardiography evaluation of intracardiac masses. Echocardiography 2006;23:218-24. 